\title{
液压阻尼孔的宽温度范围流动特性试验研究
}

\author{
王文林 $^{1}$ 㚞友权 $^{2}$ 戴谋军 $^{2}$ 陈 湘 $^{2}$ 孔 新 $^{1,3}$ 吴永明 $^{3}$ \\ (1. 东莞理工学院机械工程学院 东莞 523808; \\ 2. 株洲联诚集团减振器有限责任公司 株洲 412001; \\ 3. 广东工业大学机电工程学院 广州 510006)
}

\begin{abstract}
摘要: 研制一种适合对各种液压孔口或缝隙进行高低温流体力学试验的新型试验装置, 运用该装置对具有不同几何参数的液 压阻尼孔进行在 $-50 \sim 80{ }^{\circ} \mathrm{C}$ 宽温度范围内的流动特性试验, 研究以普通抗磨液压油 HM46 和低温抗凝减振器油 TITAN SAF 5045 为工质及其温度变化时对液压阻尼孔流量-压力特性曲线、幂指数和流量系数的影响, 研究表明, 在低温条件下, 液压 阻尼孔的流量系数均因油液秥度增大、流动性变差而呈线性下降的趋势, 从宏观上看, HM46 通过液压阻尼孔时的流动稳定 性较差, 其对应流量系数的下降幅度明显大于 TITAN SAF 5045 对应的下降幅度, 厚壁小孔流量系数的下降幅度明显大于薄 壁小孔对应的下降幅度。研究所获得的新型试验装置、试验数据分析方法和具体理论公式为深入研究和优化现代液压元件在 宽温度范围内的动态性能提供新型试验平台与理论基础。
\end{abstract}

关键词: 液压阻尼孔; 流体力学试验; 流量系数; 雷诺数; 油温

中图分类号: TH137

\section{Wide Temperature Range Experimental Study into the Fluid Mechanics of Hydraulic Orifices}

\author{
WANG Wenlin $^{1} \quad$ FAN Youquan $^{2}$ DAI Moujun ${ }^{2}$ CHEN Xiang ${ }^{2}$ \\ KONG Xin ${ }^{1,3}$ WU Yongming ${ }^{3}$
}

(1. School of Mechanical Engineering, Dongguan University of Technology, Dongguan 523808;

2. Zhuzhou Lince Group Shock Absorber Co., Ltd., Zhuzhou 412001;

3. School of Electromechanical Engineering, Guangdong University of Technology, Guangzhou 510006)

\begin{abstract}
A novel experimental equipment capable of testing the fluid mechanics of hydraulic orifices or gaps under low or high temperature conditions is developed. By using the equipment, experiments on discharge characteristics of hydraulic orifices with different geometric parameters are conducted in a wide temperature range of $-50-80{ }^{\circ} \mathrm{C}$. The effects of the common anti-wear hydraulic oil HM46 and the anti-frozen shock absorber oil TITAN SAF 5045 with different temperatures on the flow-pressure characteristics and its exponent, discharge coefficient of the orifices are then investigated. The research results show that at low temperatures, discharge coefficients of the orifices will drop linearly due to viscosity increasing and fluidity decreasing of the fluids, and in a macro sense, when with HM46 the fluidity is worse and the discharge coefficient drop is obviously greater than that when with TITAN SAF 5045, the discharge coefficient drop of the thick-walled orifice is obviously greater than that of the sharp-edged orifice. The obtained new experimental equipment, test data processing approach and concrete theoretical formulae in this study have brought a new experimental platform and the basic theory for studying and/or optimizing the dynamic performance of modern hydraulic exponents in a wide temperature range.
\end{abstract}

Key words: hydraulic orifice; fluid mechanics experiment; discharge coefficient; Reynolds number; oil temperature

\footnotetext{
* 国家自然科学基金(11572123) 和东莞理工学院高层次人才 (KCYXM2017026)资助项目。20200227 收到初稿, 20200816 收到修改稿
} 


\section{0 前言}

阻尼孔在液压技术中占有重要地位, 其出流特 性对液压元器件的动态特性起着关键作用。由于现 代高端装备越来越要求能在极端环境下安全服役, 譬如高铁油压减振器的极端服役温度范围要求达到 $-40 \sim 70{ }^{\circ} \mathrm{C}^{[1-2]}$, 因此研究液压阻尼孔在宽温度范围 内的流动特性, 对提高液压元件品质和主机系统的 环境适应性具有重要意义。

现有关于流体孔口出流特性的研究, 一般都是 默认在常温环境下进行的, 并且以水流动为基础或 参考。早期 LICHTAROWICZ 等 ${ }^{[3]}$ 运用试验方法研 究了长径比 $l / d$ 至 10 、雷诺数至 $10^{5}$ 的孔口出流的流 量系数, 测试流体包括水、水-甘油混合物和油, 并 基于试验数据分析给出了流量系数的经验公式 ${ }^{[4-5]}$, RAMAMURTHI 等 ${ }^{[6]}$ 也基于试验方法研究了软化水 流经薄壁孔口的流量系数变化规律, $\mathrm{YU}$ 等 ${ }^{[7-8]}$ 则试 验研究了背压对孔口水射流流量系数的影响; 张效 伟等 ${ }^{[9]}$ 试验了以常温压缩空气为工质的孔口流量系 数, 研究了孔口几何参数和雷诺数对气流流量系数 的影响。

运用计算流体动力学 (Computational fluid dynamics, CFD) 方法研究 ${ }^{[10-11]}$ 孔口出流特性是一种 现代方法, 但其本质上是以经典流体力学理论和试 验数据为基准的, 丁勇为 ${ }^{[12]}$ 结合 CFD 和试验方法研 究了液压阻尼孔的长径比、孔径管径比和孔口倒角 对飞机起落架缓冲器阻尼特性和落震动力学的影 响。此外, 由于多孔流动相对于传统单孔流动, 具 有明显改善流态、降低能量损失等优点, 近年来使 用多孔板作为流量计成为了一个研究热点, 工质涉 及常温水 ${ }^{[13]}$ 、城市供暖热水 ${ }^{[14]}$ 和液氮 ${ }^{[15]}$, 但研究内 容和方法与传统研究类似。

目前针对以矿物油为工质的液压阻尼孔流动特 性的研究, 一般也是默认在常温环境 ${ }^{[16]}$ 下进行的, 针对液压阻尼孔在高低温、特别是在低温条件下流 动特性的研究, 显得非常不足, 也没有相关试验设 备。为了满足现代以液压技术为基础的高端装备能 在极端环境下安全服役的需求，开展液压阻尼孔在 宽温度范围内的流动特性基础研究, 本文首先研制 了一种适合于进行液压孔口和缝隙高低温流体力学 试验的新型试验装置, 运用该装置针对常用的薄壁 小孔、厚壁小孔进行了在 $-50 \sim 80{ }^{\circ} \mathrm{C}$ 的流动特性试 验, 给出了通过试验数据拟合获得液压阻尼孔流量压力特性曲线和流量系数的方法和理论公式, 研究
了油温、不同工质对流量-压力特性曲线、幂指数和 流量系数的影响, 并分别获得了以两种液压油为工 质的液压阻尼孔在高雷诺数区间其流量系数的经验 公式, 本文研究成果为深入研究和优化液压元件在 宽温度范围内的动态性能提供了试验平台和理论 基础。

\section{1 新型试验装置的研制}

现有关于液压孔口流动特性的试验, 是在常温 环境下进行的，其试验装置一般都采用了包括液压 泵、液压阀、蓄能器等在内的一整套液压系统 ${ }^{[16]}$ 向 被试液压孔口供油的原理, 这种试验原理在高低温、 特别是在低温条件下是不可行的，因为在低温譬如 $-30{ }^{\circ} \mathrm{C}$ 以下时, 大部分液压元件如泵已不能有效工 作, 再者如果采取对整个液压系统液压油进行温度 控制的方法，显得既不方便也不节能。

借鉴铁道车辆双筒式油压减振器的主体结构形 式, 提出了一种适合于对液压孔口和缝隙在高低温 环境下进行流动特性试验的一体化试验模块 ${ }^{[17]}$, 其 试验原理如图 1a 所示。被试液压阻尼孔或缝隙被设 计在减振器的阻尼阀之中, 减振器的活塞-活塞杆组 件被外力驱动时, 相当于一个机械式供油机构向阻 尼孔供油; 采用两个压力传感器测试阻尼孔或缝隙 前后的压力 $P_{1} 、 P_{2}$, 从而可获得压差 $\Delta P=P_{1}-P_{2}$, 采用一个温度传感器测量实时进入阻尼孔或缝隙的 液压油的油温; 另外设计了一只安全阀，防止因误 动作导致驱动速度过快时, 保护试验装置。所有的 阀和传感器都被紧凑地集成在减振器的导座上 (图 1b), 因此整个一体化试验模块可以方便地被置 入一个高低温箱中开展试验。

基于该一体化试验模块, 开发了一种新型液压 孔口和缝隙高低温流体力学自动化试验装置 (图 1c)。试验装置采用了变频电机控制一个螺母丝 杆升降机构运动, 从而驱动减振器活塞杆运动, 运 动规律可通过工控机上的控制软件进行设置，一般 采用匀速或匀加速驱动方式。采用了一只位移传感 器实时测量活塞杆的位移, 因此通过换算可得活塞 杆的运动速度以及向被试液压孔口提供油液的流量 $q$, 此外在驱动机构和活塞杆之间设置了一个力传感 器用来测量驱动力。工控机通过对各种传感器的信 息处理, 可以输出在特定温度下, 液压油通过被试 液压孔口或缝隙时的流量-压力特性 $(q-\Delta P)$ 和流量系 数 $\left(C_{\mathrm{d}}\right)$ 等信息。 


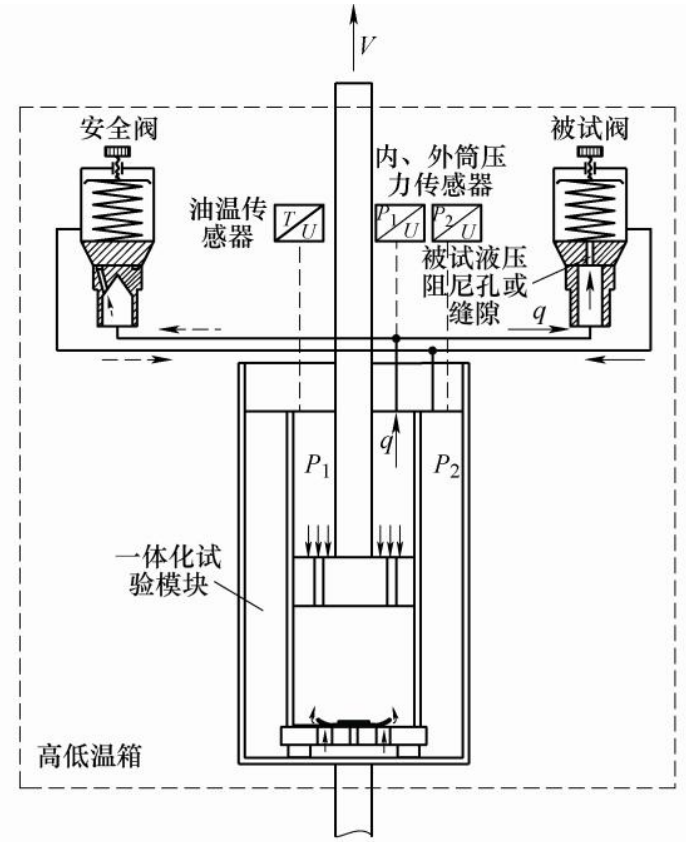

(a) 试验原理

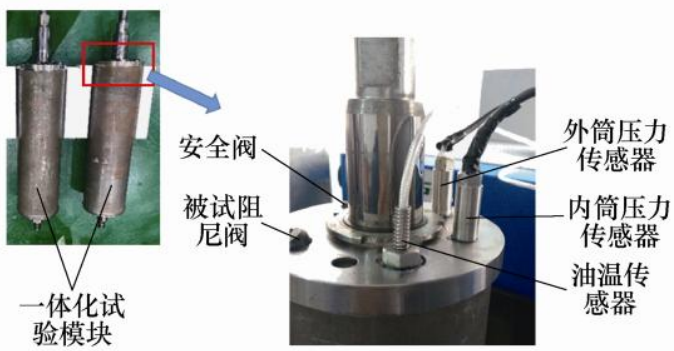

(b) 阀和传感器在一体化试验模块上的集成

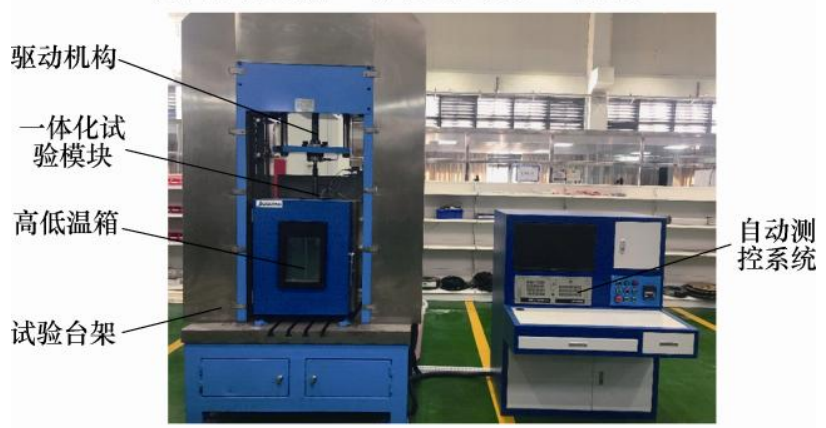

(c) 试验台总成

图 1 新型液压孔口和缝隙高低温流体力学试验装置

由于可以在被试阻尼阀中设计各种具有不同几 何参数的液压孔口或缝隙 ${ }^{[17]}$, 加上高低温箱的温度 调节范围可达-70 120 ${ }^{\circ} \mathrm{C}$, 因此该新型试验装置能 够针对常用液压孔口和缝隙进行在宽温度范围内的 流动特性测试, 是一个功能较齐全的试验平台。

\section{2 宽温度范围流动特性试验与分析}

\section{1 流动特性试验}

在液压技术 ${ }^{[5,18-19]}$ 中, 常将长径比 $l / d<0.5$ 的阻 尼孔称为薄壁小孔(或短孔), 将 $0.5<l / d<4$ 的阻尼孔
称为厚壁小孔(或阻尼长孔), 将 $l / d>4$ 的阻尼孔称为 细长孔。其中以薄壁小孔和厚壁小孔应用最为广泛, 其流量-压力特性可表述为以下幂函数

$$
q=C_{\mathrm{d}} A\left(\frac{2}{\rho} \Delta P\right)^{m}
$$

式中, $q$ 为通过阻尼孔的流量, $C_{\mathrm{d}}$ 为流量系数, $A$ 为阻尼孔截面积, $\rho$ 为油液密度, $\Delta P$ 为阻尼孔前后 压力差, $m$ 为幂指数,一般认为薄壁小孔的 $m=0.5$, 厚壁小孔 $0.5<m<1$, 在常温条件下，厚壁小孔的 $m$ 可以认为近似等于 0.5 。

为了研究油温、不同液压油对薄壁小孔和厚壁 小孔流动特性幂指数 $m$ 和流量系数 $C_{\mathrm{d}}$ 的影响, 运 用所研制的新型液压孔口高低温流体力学试验装 置, 针对表 1 所示的研究对象进行了在 $-50 \sim 80{ }^{\circ} \mathrm{C}$ 的试验, 测试温度间隔取 $10{ }^{\circ} \mathrm{C}$ 。

表 1 试验用液压阻尼孔的几何参数

\begin{tabular}{lcccc}
\hline \multirow{2}{*}{ 参数 } & \multicolumn{4}{c}{ 阻尼孔编号 } \\
\cline { 2 - 5 } & $1^{\#}$ & $2^{\#}$ & $3^{\#}$ & $4^{\#}$ \\
\hline 孔口类型 & 薄壁小孔 & 厚壁小孔 & 厚壁小孔 & 厚壁小孔 \\
直径 $d / \mathrm{mm}$ & 1.10 & 1.00 & 1.20 & 1.00 \\
长度 $l / \mathrm{mm}$ & 0.40 & 0.80 & 1.96 & 2.95 \\
长径比 $l / d$ & 0.36 & 0.80 & 1.63 & 2.95 \\
\hline
\end{tabular}

试验过程中，研制了两种注入不同工质的一体 化试验模块, 一种注入普通抗磨液压油 HM46, 其 倾点在 $-12{ }^{\circ} \mathrm{C}$ 左右, 一种注入车辆减振器油(低温抗 凝)TITAN SAF 5045, 其倾点为 $-57{ }^{\circ} \mathrm{C}$ 。选择这两 种代表性工质的目的, 是想研究倾点范围不同的液 压油在宽温度范围内通过液压阻尼孔时的流动特性 有何具体不同。此外, 在这两种一体化试验模块中, 通过更换不同的被试阻尼阀，就可以针对不同几何 参数的液压阻尼孔进行试验, 每次试验前确保一体 化试验模块在某温度点保温 $24 \mathrm{~h}^{[1]}$ 以上。

作为示例, 图 2 给出了长径比为 2.95 的厚壁小 孔通过 $-50{ }^{\circ} \mathrm{C}$ 的 TITAN SAF 5045 减振器油时的试 验数据及其拟合曲线。

忽略密度随温度的变化, 对图 2a 所示的流量压力特性试验数据, 按照式(1)的规律进行拟合得

$$
q=2.71 \times 4-5 \Delta P^{0.52}
$$

可见幂指数 $m=0.5205$; 对图 $2 \mathrm{~b}$ 所示的流量系 数试验数据采用 4 项高斯方程进行拟合得

$$
C_{\mathrm{d}}=\sum_{i=1}^{4}\left\{a_{i} \exp \left[-\left(\frac{R_{\mathrm{e}}-b_{i}}{c_{i}}\right)^{2}\right]\right\}
$$


式中, $R e$ 为表征流体通过阻尼孔流态的雷诺数, 方 程系数 $a_{i}(i=1 \sim 4)=[0.273 \quad 3,-0.0560,0.0582$, $0.6085], b_{i}(i=1 \sim 4)=[24.54,30.33,38.78,-814.6]$, $c_{i}(i=1 \sim 4)=[6.425,2.481,12.01,4624]$ 。

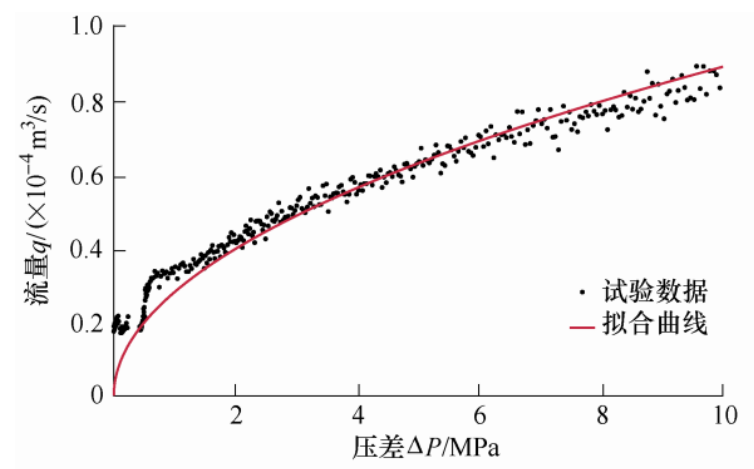

(a) 测试流量-压力特性及其拟合曲线

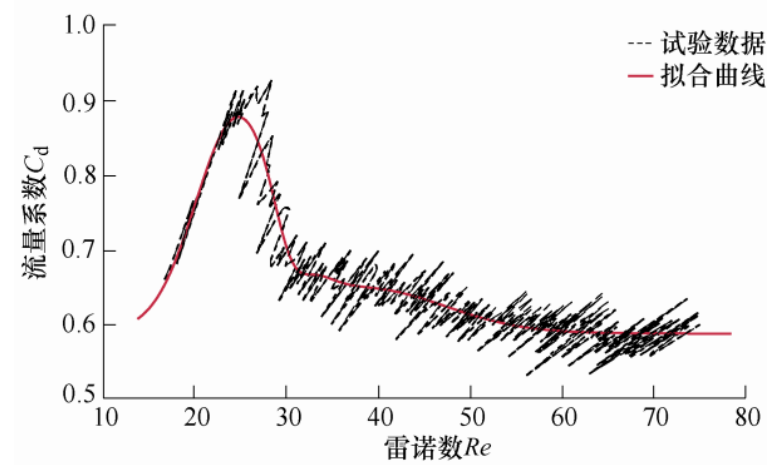

(b) 测试流量系数及其拟合曲线

图 2 液压阻尼孔的流动特性试验数据及其拟合曲线 (试验条件: 厚壁小孔, 长径比 $l / d=2.95$, 温度 $\theta=-50{ }^{\circ} \mathrm{C}$, 工质 TITAN SAF 5045)

由于圆管的水力直径就是其直径, 再根据流量 连续性方程, 可得式(3)中 $R e$ 的计算公式为

$$
R e=\frac{v_{0} d}{v}=\frac{V\left(D^{2}-d_{\mathrm{r}}^{2}\right)}{v d}
$$

式中, $v_{0}$ 和 $V$ 分别为阻尼孔内液压油流速和活塞杆 运动速度, $d 、 d_{\mathrm{r}}$ 和 $D$ 分别为阻尼孔、活塞杆和活 塞直径; $v$ 为液压油运动黏度, 前期已针对试验油液 的运动黏度等物理特性在 $-50 \sim 80{ }^{\circ} \mathrm{C}$ 进行了试验测 试和理论研究, 由于篇幅限制, 此处不再详述。

\section{2 油温和工质对阻尼孔流量-压力特性 $(q-\Delta P)$ 的影响}

作为示例, 图 3 中分别给出了一种薄壁小孔、 一种厚壁小孔在不同温度点、分别通过不同工质时 的流量-压力特性曲线的对比。由图 3a 和图 3b 可见, 薄壁小孔的流量-压力特性对油温变化以及不同工 质确实不太敏感; 由图 3c 可见, 厚壁小孔在通过低 温抗凝液压油时, 其流量-压力特性受油温变化的影 响不明显, 但在通过普通抗磨液压油时, 受油温变 化的影响较为明显(图 3d)。

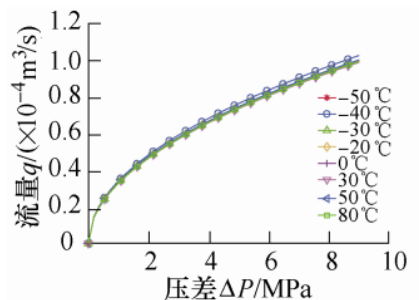

(a) $l / d=0.36 、$ TITAN SAF 5045

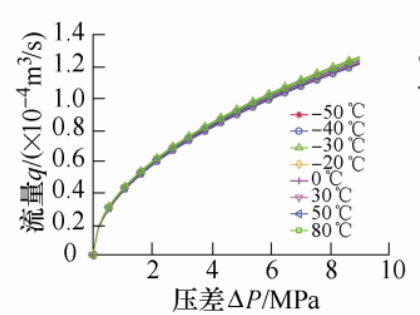

(c) $l / d=1.63$ 、TITAN SAF 5045

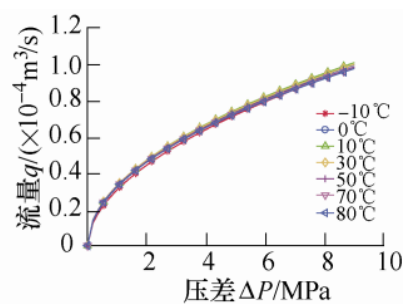

(b) $l / d=0.36 、 \mathrm{HM} 46$

图 3 油温和工质对液压阻尼孔流量-压力特性的影响

图 4 给出了四种阻尼孔在宽温度范围内、分别 通过不同工质时其流量-压力特性曲线幂指数 $m$ 变 化的统计结果。

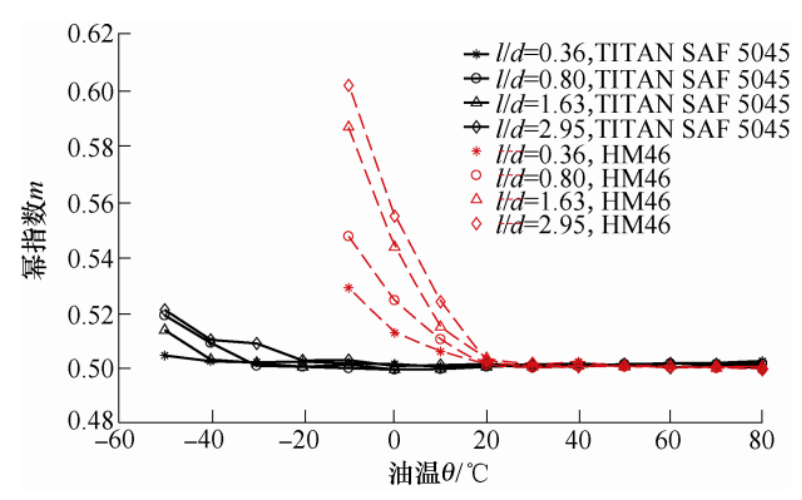

图 4 油温和工质对液压阻尼孔流量-压力 特性幂指数 $m$ 的影响

(1) 从宏观上看, 在 $20 \sim 80{ }^{\circ} \mathrm{C}$, 两种液压油在 通过各种阻尼孔时的流动特性比较稳定, 幂指数 $m$ 基本保持在 0.5 左右, 但在 $20{ }^{\circ} \mathrm{C}$ 以下到低温区间, TITAN SAF 5045 通过各种阻尼孔时的流动特性变 化, 明显小于 HM46 相应的变化。

(2) TITAN SAF 5045 通过薄壁小孔时, 在 -50 $80{ }^{\circ} \mathrm{C}$ 流动特性基本稳定, 在通过厚壁小孔时, 在 $-20 \sim 80{ }^{\circ} \mathrm{C}$ 流动特性稳定, 但在 $-50 \sim-20{ }^{\circ} \mathrm{C}$, 随 着温度下降, $m$ 呈小幅上升的趋势, 表明在相同压 差下，通过阻尼孔的流量将小幅下降。

(3) 在 $-10 \sim 20{ }^{\circ} \mathrm{C}, \mathrm{HM} 46$ 通过各种阻尼孔时, 随着温度下降, $m$ 的上升趋势比较明显(通过薄 壁小孔时, 上升趋势相对缓和), 这表明随着温 度下降, 通过阻尼力的流量将有明显下降, 由此 可见 HM46 不太适合于在低于常温的环境中 工作。 


\section{3 油温和工质对阻尼孔流量系数 $\left(\boldsymbol{C}_{\mathrm{d}}\right)$ 的影响}

图 5 中显示了油温和不同工质对某薄壁小孔和某 厚壁小孔在宽雷诺数范围内其流量系数 $C_{\mathrm{d}}$ 的影响。由 图 5a 可见, 普通抗磨液压油 HM46 在其最可能服役的 $-10 \sim 60{ }^{\circ} \mathrm{C}$ 油温, 通过薄壁小孔时的流量系数在 $0.47 \sim 0.91$ 变化; 在高温 $60{ }^{\circ} \mathrm{C}$ 时, 由于运动黏度比正 常温度 $30{ }^{\circ} \mathrm{C}$ 时下降了, 因此在相同流体流速范围内 其雷诺数范围扩大了, 在相同流体流速下, $60{ }^{\circ} \mathrm{C}$ 时的 $C_{\mathrm{d}}$ 略高于 $30{ }^{\circ} \mathrm{C}$ 时的 $C_{\mathrm{d}}$; 在低温 $-10{ }^{\circ} \mathrm{C}$ 时, 由于运动 黏度急剧增大, HM46 变成了黏稠状的流体(其倾点在 $-12{ }^{\circ} \mathrm{C}$ 左右), 流动性变得较差, 一是导致雷诺数大幅 下降, 二是导致 $C_{\mathrm{d}}$ 明显减小, 表明在低温条件下 $\mathrm{HM} 46$ 通过薄壁小孔的通流能力明显变差。

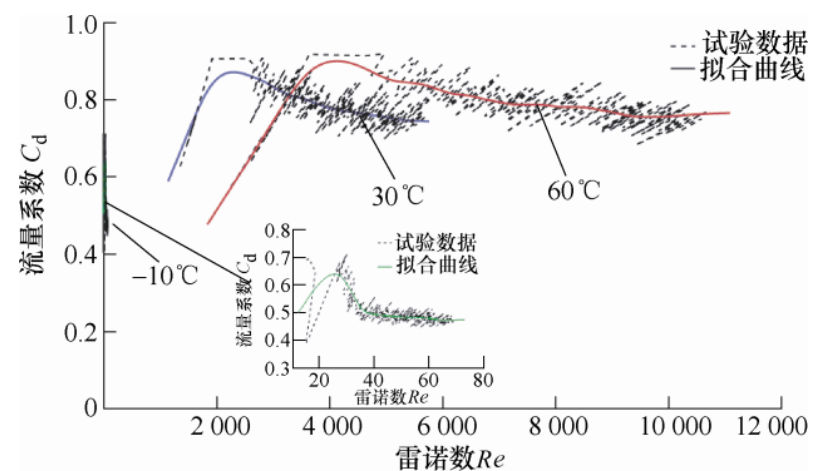

(a) $l / d=0.36$, HM46

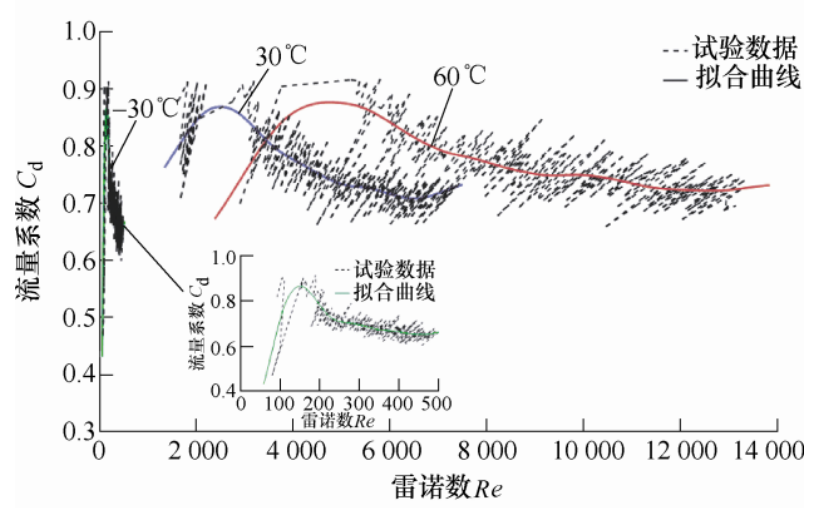

(b) $l / d=2.95$, TITAN SAF 5045

图 5 油温和工质对液压阻尼孔宽雷诺数范围流量 系数 $C_{\mathrm{d}}$ 的影响

由图 5b 可见, 车辆减振器油 TITAN SAF 5045 在其最可能服役的 $-30 \sim 60{ }^{\circ} \mathrm{C}$ 油温, 通过厚壁小孔 时的流量系数的变化规律与图 5a 所示的规律类似, 但由于 TITAN SAF 5045 是一种高性能的低温抗凝 液压油, 在 $-30{ }^{\circ} \mathrm{C}$ 的条件下, 其流量系数的下降幅 度并不显著。

图 6 显示了具有不同长径比的液压阻尼孔, 在相同 油温、相同工质条件下其 $C_{\mathrm{d}}$ 变化规律的对比。由图 $6 \mathrm{a}$ 可见, 在正常工作温度 $30{ }^{\circ} \mathrm{C}$ 时, HM46 通过各种阻尼 孔时 $C_{\mathrm{d}}$ 变化规律存在小的差异性, 在高温 $80{ }^{\circ} \mathrm{C}$ 时差异 性减小(图 6b); 从宏观上看, 阻尼孔的长径比增加, 其
$C_{\mathrm{d}}$ 有减小的趋势, 这种趋势在低温条件下(图 6c), 显得 非常明显, 表明随着长径比增加, 流体通过阻尼孔时的 能量损失加大, 通流能力下降。

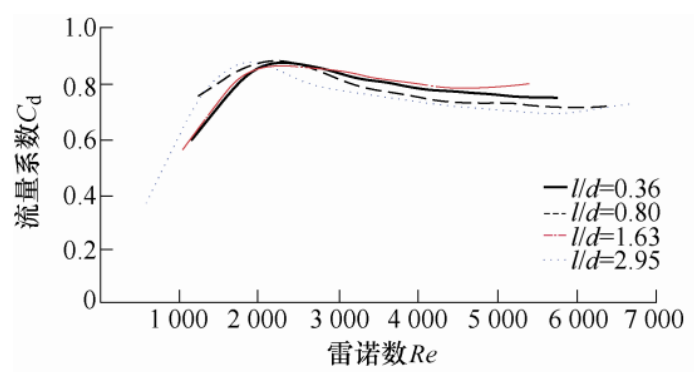

(a) $\mathrm{T}=30^{\circ} \mathrm{C}, \mathrm{HM} 46$

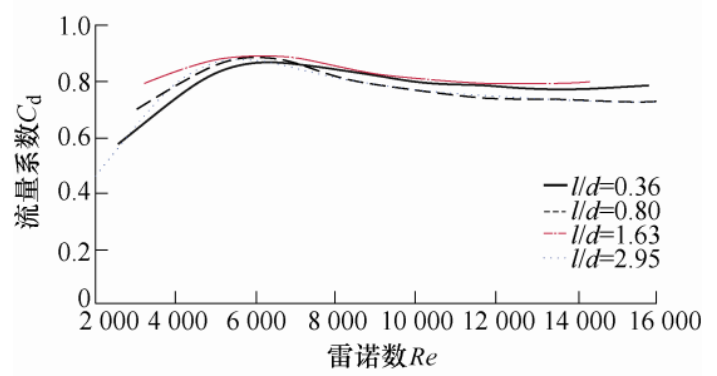

(b) $T=80^{\circ} \mathrm{C}, \mathrm{HM} 46$

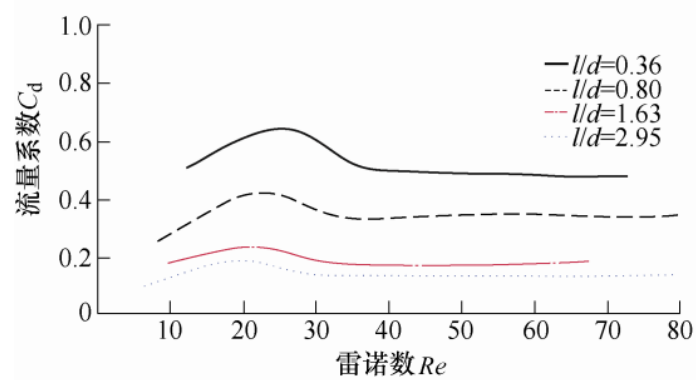

(c) $T=-10^{\circ} \mathrm{C}, \mathrm{HM} 46$

图 6 相同条件下不同长径比液压阻尼孔的流量系数 $C_{\mathrm{d}}$

图 7、8 中综合统计了 TITAN SAF 5045 和 HM46 液压油, 在宽温度范围内通过各种阻尼孔时, 在高 雷诺数区间其流量系数 $C_{\mathrm{d}}$ 的数值。由图 7、8 总体 可见, 薄壁小孔的 $C_{\mathrm{d}}$ 比厚壁小孔的 $C_{\mathrm{d}}$ 相对稳定, TITAN SAF 5045 的 $C_{\mathrm{d}}$ 比 HM46 的 $C_{\mathrm{d}}$ 相对稳定, 其 工作温度范围和环境适应性明显优于 HM46。

根据图 7 的统计数据, 推导出 TITAN SAF 5045 车辆减振器油, 在宽温度范围内其高雷诺数区间流 量系数 $C_{\mathrm{d}}$ 的经验公式如下

$$
C_{\mathrm{d}}=\left\{\begin{array}{rll}
0.794+0.005 \frac{l}{d}+0.006 T & \\
T \in\left[-50{ }^{\circ} \mathrm{C},-25^{\circ} \mathrm{C}\right] & 0.5<\frac{l}{d}<4 \\
0.689 & T \in\left(-25^{\circ} \mathrm{C}, 80^{\circ} \mathrm{C}\right] & 0.5<\frac{l}{d}<4 \\
0.703 & T \in\left[-50^{\circ} \mathrm{C}, 80^{\circ} \mathrm{C}\right] & \frac{l}{d}<0.5
\end{array}\right.
$$

式中, $T$ 为油温。 


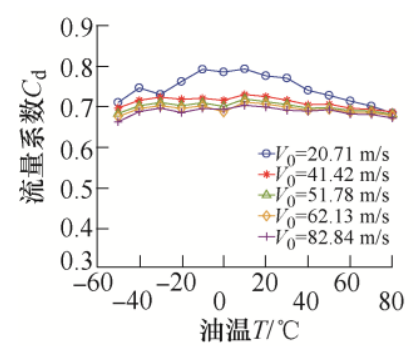

(a) $l / d=0.36$

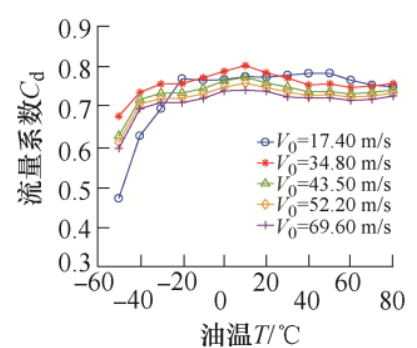

(c) $l / d=1.63$

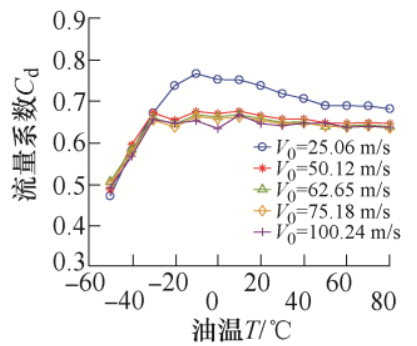

(b) $l / d=0.8$

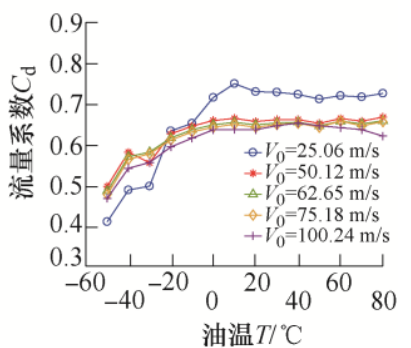

(d) $l / d=2.95$
图 7 油温和工质对液压阻尼孔高雷诺数区间流量系数 $C_{\mathrm{d}}$ 的影响 (工质: TITAN SAF 5045)

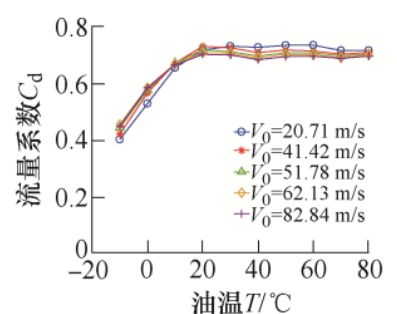

(a) $l / d=0.36$

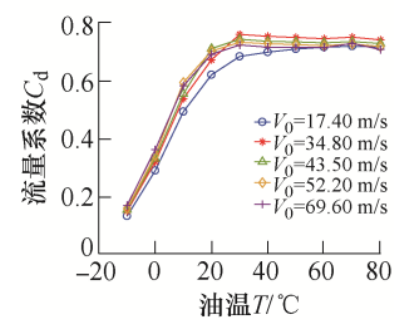

(c) $l / d=1.63$

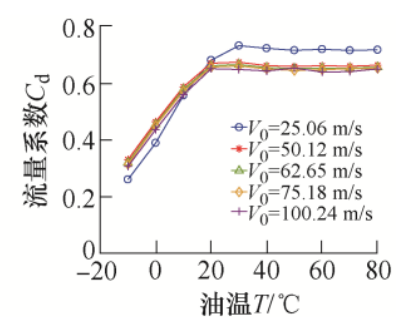

(b) $l / d=0.8$

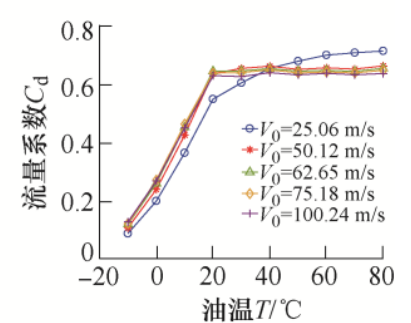

(d) $l / d=2.95$
图 8 油温和工质对液压阻尼孔高雷诺数区间流量

系数 $C_{\mathrm{d}}$ 的影响(工质: HM46)

同理, 根据图 8 的统计数据, 推导出 HM46 液 压油, 在宽温度范围内其高雷诺数区间流量系数 $C_{\mathrm{d}}$ 的经验公式如下

$$
C_{\mathrm{d}}=\left\{\begin{array}{rr}
0.625-0.315 \frac{l}{d}+0.011 T \\
T \in\left[-10{ }^{\circ} \mathrm{C}, 20^{\circ} \mathrm{C}\right] & \frac{l}{d}<1.5 \\
0.401-0.046 \frac{l}{d}+0.017 T & \\
T \in\left[-10{ }^{\circ} \mathrm{C}, 20^{\circ} \mathrm{C}\right] & 1.5<\frac{l}{d}<4 \\
0.685 \quad T \in\left(20^{\circ} \mathrm{C}, 80^{\circ} \mathrm{C}\right] & \frac{l}{d}<4
\end{array}\right.
$$

经验公式(5)、(6)基于试验数据获得, 综合考虑
了油温和阻尼孔长径比因素, 如果仅用于工程估算, 其精度是可以满足要求的。但在研究和优化使用以 上两种液压油的液压元件性能时, 计算精度要求很 高, 就应根据每一个液压阻尼孔的几何参数、油温, 有针对性地参照式(1) (4) 的理论进行拟合和获得 具体数据。

\section{3 结论}

（1）研制了一种基于一体化试验模块的高低温 流体力学新型试验装置, 由于可以在其一体化试验 模块的被试阻尼阀中, 设计不同几何参数的液压孔 口或缝隙来模拟各种孔口流动或缝隙流动, 加上其 高低温箱的温度调节范围可达 $-70 \sim 120{ }^{\circ} \mathrm{C}$, 因此该 新型试验装置能够针对常用液压孔口或缝隙进行在 宽温度范围内流动特性的测试, 形成了一个功能较 齐全的试验平台。

(2) 薄壁小孔的流量-压力特性对不同液压油及 其油温变化不太敏感: 在宽温度范围内通过低温抗 凝液压油、以及在常温范围内通过普通抗磨液压油 时, 其幂指数 $m=0.5$, 但在低温范围内通过普通抗 磨液压油时, 其幂指数会有小幅上升, 在 $0.50 \sim 0.53$ 变化; 厚壁小孔在通过低温抗凝液压油时, 其流量压力特性幂指数 $m$ 在接近油液倾点的低温区间有小 幅上升, 但不明显, 但在通过低于常温的普通抗磨 液压油时, 其流动特性受油温变化的影响较为明显, 幂指数 $m$ 在 $0.5 \sim 0.6$ 变化。因此在低温条件下, 普 通抗磨液压油通过液压阻尼孔时的流动稳定性较 差, 会引起压力、流量与设计值偏离较大, 不太适 合于在低于常温的环境中工作。

(3) 在常温条件下, 液压阻尼孔长径比增加, 对应的流量系数略下降; 在高温区间, 液压阻尼孔 的流量系数略高于其常温下的流量系数, 并且长径 比变化对其影响减弱; 但在低温条件下(低温抗凝液 压油接近倾点的区间、普通液压油低于常温的区 间), 液压阻尼孔的流量系数(即通流能力)均因油液 黏度增大、流动性变差而呈线性下降的趋势, 从宏 观上看, 普通液压油对应流量系数的下降幅度明显 大于低温抗凝液压油对应的下降幅度, 厚壁小孔流 量系数的下降幅度明显大于薄壁小孔对应的下降 幅度。

(4) 研究所获得的新型试验装置、试验数据分 析方法和具体理论公式, 为继续深入研究和优化 ${ }^{[2,20]}$ 现代液压元件在宽温度范围内的动态性能提供了新 型试验平台与理论基础。 


\section{参 考 文 献}

[1] European Committee for Standardization. EN13802: 2013 railway applications - suspension components - hydraulic damper[S]. Brussels : CEN-CENELEC Management Centre, 2013.

[2] WANG Wenlin, LIANG Yuwen, ZHANG Weihua, et al. Experimental research into the low-temperature characteristics of a hydraulic damper and the effect on the dynamics of the pantograph of a high-speed train running in extreme cold weather conditions[J]. Proceedings of the Institution of Mechanical Engineers, Part F: Journal of Rail and Rapid Transit, 2020， 234(8): 896-907.

[3] LICHTAROWICZ A, DUGGINS R K, MARKLAND E. Discharge coefficients for incompressible non-cavitating flow through long orifices[J]. Journal of Mechanical Engineering Science, 1965, 7(2): 210-219.

[4] MERRITT H E. Hydraulic control systems[M]. New York: John Wiley \& Sons, Inc., 1967.

[5] 许耀铭. 油膜理论与液压葲和马达的摩擦副设计 [M]. 北京: 机械工业出版社, 1987.

XU Yaoming. Thin-film theory for friction pair design of hydraulic pump and motor[M]. Beijing: China Machine Press, 1987.

[6] RAMAMURTHI K, NANDAKUMAR K. Characteristics of flow through small sharp-edged cylindrical orifices[J]. Flow Measurement and Instrumentation, 1999, 10: 133143.

[7] YU B, FU P F, ZHANG T, et al. The influence of back pressure on the flow discharge coefficients of plain orifice nozzle[J]. International Journal of Heat and Fluid Flow, 2013, 44: 509-514.

[8] THARAKAN $\mathrm{T} \mathrm{J}$, RAFEEQUE $\mathrm{T}$ A. The role of backpressure on discharge coefficient of sharp edged injection orifices[J]. Aerospace Science and Technology, 2016, 49: 269-275.

[9] 张效伟, 朱惠人, 张霞, 等. 小尺度孔的流量系数试 验[J]. 航空动力学报, 2010, 25(11): 2479-2485.

ZHANG Xiaowei, ZHU Huiren, ZHANG Xia, et al. Experiment of discharge coefficients for small scale cylindrical holes[J]. Journal of Aerospace Power, 2010, 25(11): 2479-2485.

[10] 范海平, 曾小林. 基于计算流体动力学的流量系数研 究[J]. 机电设备, 2007, 24(2): 9-12.

FAN Haiping, ZENG Xiaolin. Research of discharge coefficient based on computing fluid dynamics[J]. Mechanical and Electrical Equipment, 2007, 24(2): 9-12.

[11] NAVEENJI A, MALAVARAYAN S, KAUSHIK M, et al. CFD analysis on discharge coefficient during non-Newtonian flows through orifice meter[J]. International Journal of Engineering Science and Technology, 2010, 2(7): 3151-3164.

[12] 丁勇为. 起落架缓冲器节流装置阻尼特性研究[D]. 南 京: 南京航空航天大学, 2018.

DING Yongwei. Study on damping performance of landing gear shock absorber throttle device[D]. Nanjing: Nanjing University of Aeronautics and Astronautics, 2018.

[13] HUANG Shanfang, MA Taiyi, WANG Dong. Study on discharge coefficient of perforated orifices as a new kind of flowmeter[J]. Experimental Thermal and Fluid Science, 2013, 46: 74-83.

[14] 刘畅. 特定安装条件下孔板流量传感器流出系数的研 究[D]. 哈尔滨：哈尔滨工业大学, 2018.

LIU Chang. Research on discharge coefficient of orifice plate flow sensor under specific installation conditions[D]. Harbin: Harbin Institute of Technology, 2018.

[15] 田红. 应用于低温流体的多孔板流量计特性研究 [D]. 杭州: 浙江大学, 2016 .

TIAN Hong. Performance study on the perforated plate flowmeter applied to cryogenic fluid[D]. Hangzhou: Zhejiang University, 2016.

[16] 刘欢, 李长亮, 贾瑞清. 薄壁小孔孔口节流试验平台的 设计 $[\mathrm{J}]$. 机电产品开发与创新，2012，25(5): 41-43.

LIU Huan, LI Changliang, JIA Ruiqing. The design of the thin-walled orifice throttling test platform[J]. Development \& Innovation of Machinery \& Electrical Products, 2012, 25(5): 41-43.

[17] 王文林, 周梓荣, 曹晓畅, 等. 一种用于液压孔口和/ 或缝隙高、低温流体力学实验的一体化实验模块: 中 国，201710825931.9[P]. 2019-05-21.

WANG Wenlin, ZHOU Zirong, CAO Xiaochang, et al. A compact experimental module for hydraulic orifices and/or gaps fluid mechanics testing under both high and low temperature conditions: China, 201710825931.9[P]. 2019-05-21.

[18] 盛敬超. 液压流体力学 $[\mathrm{M}]$. 北京: 机械工业出版社, 1980.

SHENG Jingchao. Fluid mechanics for fluid power engineering[M]. Beijing: China Machine Press, 1980. 
[19] 吴根茂, 邱敏秀, 王庆丰, 等. 新编实用电液比例技 术[M]. 杭州: 浙江大学出版社, 2006.

WU Genmao, QIU Minxiu, WANG Qingfeng, et al. Electrohydraulic proportional technique in theory and application[M]. Hangzhou: Zhejiang University Press, 2006.

[20] WANG Wenlin, LIANG Yuwen, ZHANG Weihua, et al.
Effect of the nonlinear displacement-dependent characteristics of a hydraulic damper on high-speed rail pantograph dynamics[J]. Nonlinear Dynamics, 2019, 95(4): 3439-3464.

作者简介: 王文林(通信作者), 男, 1969 年出生, 博士, 教授。主要研 究方向为载运工具振动与控制、航天运载流体技术与可靠性。

E-mail: pianowwl@vip.163.com 